\title{
8
}
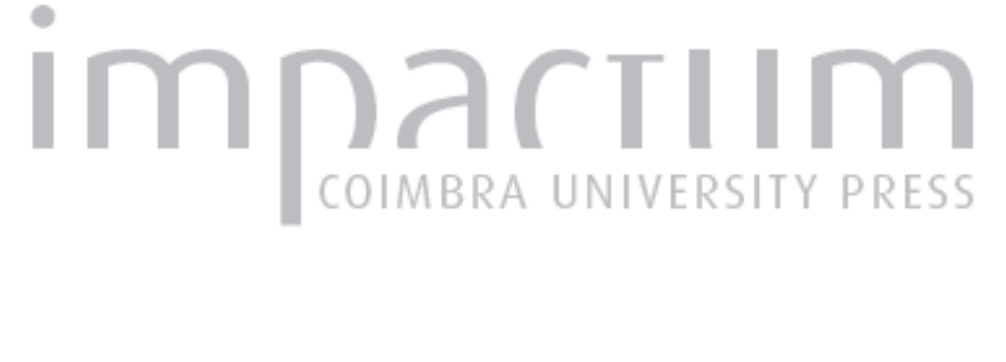

\section{Della guerra e della malattia: la memoria letteraria contro l'attenuazione della coscienza}
Autor(es):
Marco, Rosaria de

Publicado por: Imprensa da Universidade de Coimbra

URL persistente:

URI:http://hdl.handle.net/10316.2/42649

DOI:

DOI:https://doi.org/10.14195/0870-8584_2_15

Accessed : $\quad$ 26-Apr-2023 13:13:06

A navegação consulta e descarregamento dos títulos inseridos nas Bibliotecas Digitais UC Digitalis, UC Pombalina e UC Impactum, pressupõem a aceitação plena e sem reservas dos Termos e Condições de Uso destas Bibliotecas Digitais, disponíveis em https://digitalis.uc.pt/pt-pt/termos.

Conforme exposto nos referidos Termos e Condições de Uso, o descarregamento de títulos de acesso restrito requer uma licença válida de autorização devendo o utilizador aceder ao(s) documento(s) a partir de um endereço de IP da instituição detentora da supramencionada licença.

Ao utilizador é apenas permitido o descarregamento para uso pessoal, pelo que o emprego do(s) título(s) descarregado(s) para outro fim, designadamente comercial, carece de autorização do respetivo autor ou editor da obra.

Na medida em que todas as obras da UC Digitalis se encontram protegidas pelo Código do Direito de Autor e Direitos Conexos e demais legislação aplicável, toda a cópia, parcial ou total, deste documento, nos casos em que é legalmente admitida, deverá conter ou fazer-se acompanhar por este aviso.

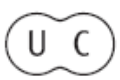




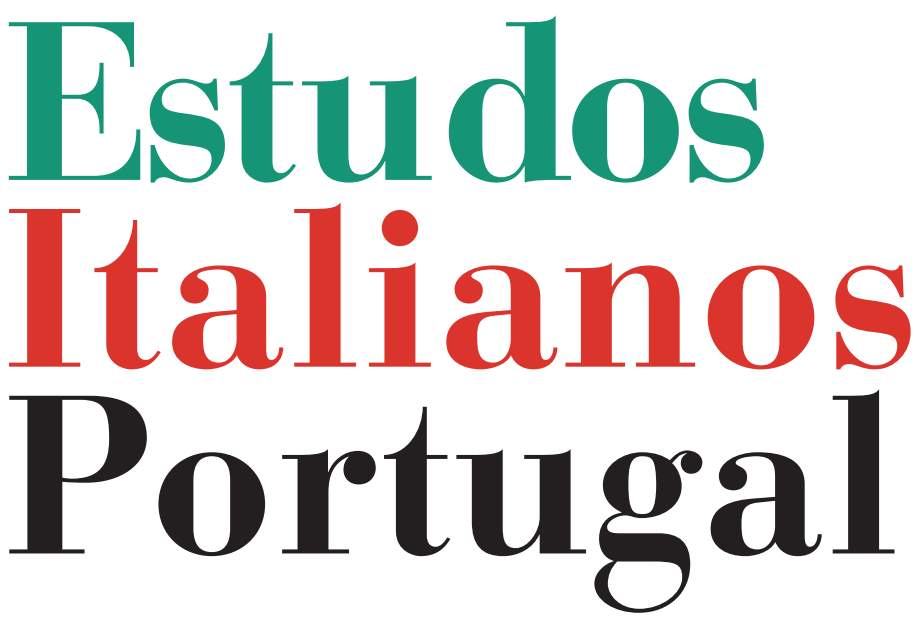

Instituto

Italiano

de Cultura

de Lisboa

Nova Série

$\mathbf{N}^{\mathbf{0}} 2$ 


\section{DELLA GUERRA E DELLA MALATTIA, LA MEMORIA LETTERARIA CONTRO L'ATTENUAZIONE DELLA COSCIENZA}

Rosaria DE MARCO*

Nell'introduzione di UNA EDIZIONE italiana de L'idiota $^{1}$ di Dostoevskij, Mauro Martini scrive: “...Myškin, incurante del disagio di chi gli sta di fronte, punta semmai a mettere a nudo il nucleo del dolore dei suoi interlocutori. E, ciò facendo, travolge le difese che costoro si sono faticosamente eretti per proteggersi dal contatto diretto con il pulsare del loro male interiore.[...] E propone un ambito radicalmente nuovo. Non importa amare o odiare, fare il bene o il male, se ciò conduce inesorabilmente a commettere l'unico vero peccato, che è quello di distrarsi dal prossimo..."2. Questa interpretazione mi sembra trasferibile alla funzione che Diceria dell'untore di Gesualdo Bufalino e Os cus de Judas di António Lobo Antunes hanno avuto nelle rispettive culture e nella sensibilità dei lettori. Il loro comportamento è, infatti,

* Rosaria de Marco è dottoranda in Letterature Romanze Comparate all'Università di Napoli L'Orientale e collaboratrice della Cattedra di Letteratura Portoghese dell'Università Suor Orsola Benincasa. Ha tradotto i racconti di A. Herculano e di M.de Sá-Carneiro in Quando il diavolo ci mette la coda, Napoli, L'Ancora de Mediterraneo, 2006. Suoi contributi sono presenti in diversi volumi collettivi. Ha pubblicato il romanzo Il gioco della luna e del vento (Napoli, OXP, 2006).

1 M.Martini, "Introduzione”, in F.M. Dostoevskij, L'idiota, Roma, Newton Compton, 2002, pp. 7-8.

2 Ibidem, p. 8.

Est.Ital.Port., n.s., 2, 2007: 305-324 
improntato alla violazione della norma-lità (estetica e sociale) come quello di Myškin e, come quello, risulta essere strategico al perseguimento di un fine analogo: l'incontro tra due pulsioni primigenie, "il dolore radicale dell'uno e la disponibilità immediata dell'altro ad assumerlo"3, il dolore testimoniato dal romanzo, la disponibilità del lettore a sentirlo. La peculiarità di queste due opere e il motivo che mi ha indotta a questa riflessione sta nella loro capacità di opporsi al rischio che "la paura di ciascuno per sé, arrivi ad assorbire lo scandalo dell'indifferenza alla sofferenza altrui" "

1) La Diceria dell'untore è un romanzo della memoria che racconta, in prima persona, la convivenza di alcuni malati terminali di TBC in un sanatorio siciliano nel 1946. Tra il protagonista ed una paziente nasce una storia d'amore agonica che sfocia in una insensata fuga a due, subito interrotta dalla morte di lei. "Egli guarisce, invece, inaspettatamente, e rientrando nella vita di tutti i giorni, vi porta un'educazione alla catastrofe di cui probabilmente non saprà servirsi, ma anche la ricchezza di un noviziato indimenticabile nel reame delle ombre"s.

Il materiale trattato è disagevole, scabroso per la miseria umana che scandaglia con una vicinanza pericolosa, quasi infetta. Le parole ${ }^{6}$, sempre un po' al di qua o un po' al di là della realtà, sono spesso più crudeli della realtà stessa per la necessità di compensare la non esistenza. Sono testimonianza di un universo di frontiera, di confine estremo tra la vita e la morte. Un territorio percorso per secoli da eroi tratteggiati a sfolgoranti colpi di retorica, qui viene, invece, attraversato sommessamente da protagonisti della sconfitta, una

3 Ibidem, p. 9.

${ }^{4}$ E. Lévinas, Tra noi, Milano, Jaka Book, 1998, p. 227.

${ }^{5}$ G.Bufalino, Diceria dell'untore, $1^{\mathrm{a}}$ ed., Palermo, Sellerio 1981 (7 ${ }^{\mathrm{a}} \mathrm{ed}$., Milano, Bompiani 1997), p. 179.

${ }^{6} \mathrm{Mi}$ sembra qui significativo richiamare l'etimologia di parola: dal latino parabola $(m)$ che deriva a sua volta dal greco e significa avvicinamento, paragone. 
sconfitta tanto più umiliante perché inflitta dal nemico invisibile e interno di una malattia infamante. Schegge di umanità insufficienti che abbandonano la scena comune in punta di piedi, occultando la propria vergogna nell'esilio volontario di un sanatorio, tra pari, confortati dall'inconfessabile vantaggio di non essere esposti alla morte più di quanto lo siano tutti coloro che li circondano.

Per l'epoca (1981), la prosa della Diceria dell'untore era decisamente troppo turgida, esuberante di artifici retorici, intenzionata a sfidare le tendenze contemporanee di asciuttezza ed essenzialità con una spregiudicata estensione di intonazioni, fino al melodramma, appena corrette da un accorto impiego di ironia. Nonostante il successo di critica e di pubblico che accolse questo esordio tardivo, consacrato dalla vittoria del premio Campiello nello stesso anno della pubblicazione, sembra non essere un romanzo molto letto.

Anche Os cus de Judas dello scrittore portoghese António Lobo Antunes è un romanzo della memoria, sulla "dolorosa aprendizagem da agonia" 7 in cui tutto quanto accade è in relazione all'Io interiore del protagonista che usurpa l'autore testuale fino all'indistinzione. Il romanzo è un lungo, disperato monologo di reduce della guerra coloniale portoghese. Spedito in Angola come molti altri giovani oppositori di cui il regime salazarista si liberava in questo modo, rientra in patria sperimentando un'umiliante estraneità: "Flutuo entre dois continentes que me repelem..."

Rifugiato nell'irrealtà della notte in un bar di Lisbona, disinibito dall'alcol, tormentato dai ricordi, apparentemente ansioso di colmare i silenzi della sua occasionale, muta, compagna e scardinare l'indifferenza che le legge negli occhi

7 A. Lobo Antunes, Os cus de Judas, $1^{\text {a }}$ ed., Lisboa, 1979 ( 20 ${ }^{\text {a }}$, Lisboa, Dom Quixote, 2000, p. 43).

${ }^{8}$ A. Lobo Antunes, ibidem, p. 226. 
(che è l'indifferenza stessa del suo Paese), il protagonista produce un discorso d'intonazione furiosa, appena attenuato da divagazioni continue e da una vena di ironia che lo attraversa tutto, e agitato dalla febbrile intermittenza tra passato e presente. Probabilmente, ciò che veramente cerca di costruire con il suo oltranzismo verbale è un ponte che lo riporti verso gli altri, lontano dalla propria solitudine. $\mathrm{O}$ che porti qualcuno verso di lui. Luciana Stegagno Picchio, nella recensione di Os cus de Judas appena pubblicato in Italia, parlò dell"“espressionismo barocco di questa prosa".

Alla sua prima edizione in Portogallo, nel 1979, il romanzo di Lobo Antunes suscitò reazioni imbarazzate oltre che per la prosa violentemente espressionista, per l'argomento traumatico e scabroso e, pertanto, soggetto più facilmente ad un trattamento di rimozione che di elaborazione. Os cus de Judas si proponeva, in maniera radicale, come parola contro l'impunità del silenzio.

2) Secondo Italo Calvino l'incipit svolge una funzione fondamentale perché rappresenta il "distacco dalla molteplicità dei possibili" ${ }^{10}$, necessario al narratore per definire la sua storia isolandola, estraendola dalla moltitudine di storie. L'incipit è "il luogo letterario per eccellenza" quanto passaggio dal mondo delle cose e dei fatti a quello delle parole e da quello di tutte le possibili parole alle parole dell'autore. È una zona di confine, dunque, dove sono osservabili i modi in cui l'operazione letteraria costruisce la propria riflessione su qualcosa che è altro da sé, ma che solo la letteratura può esprimere. Tenterò, dunque, una osservazione proprio delle pagine iniziali dei due romanzi, scomponendole in unità brevi per comodità di analisi.

9 Cito dall'articolo di L.Stegagno Picchio "Vi scrivo dal paese ferito", La Repubblica del 26.11.03.

10 I. Calvino, Lezioni americane, Milano, Mondadori, 2002, p. 138.

11 I. Calvino, ibidem, p. 138. 
L'incipit della Diceria dell'untore evoca un'allusione allo shakespeariano "morire, dormire, sognare forse".

O quando tutte le notti - per pigrizia, per avarizia - ritornavo a sognare lo stesso sogno: una strada color cenere, piatta, che scorre con andamento di fiume tra due muri più alti della statura di un uomo; poi si rompe, strapiomba sul vuoto.

La prima parola è una congiunzione con valore disgiuntivo che già da sola prefigura un territorio di confine; la seconda è un avverbio di tempo, testimone qui di una distanza cronologica che è anche elemento metatestuale: lo stesso Bufalino, infatti, ci informa di aver iniziato una prima stesura nel dopoguerra "della glaciazione neorealista" 12 , di averla presto interrotta perché insoddisfatto della forma espressiva che gli appariva inadeguata, e di averla, infine, ripresa intorno al '70, impegnandosi in una revisione continua e nevrotica, fino alla pubblicazione.

Due causali negative precedono il soggetto sottinteso, conferendogli, preliminarmente, un carattere di inadeguatezza e inettitudine fisiche che, con ragionevole approssimazione, costituiscono anche una sintesi del giudizio morale che l'autore conserva di quel sé remoto. Lo scenario del sogno conferma l'iniziale suggestione del limen, grigio, delimitato da alti muri laterali che escludono ogni possibile orizzonte; la nozione stessa di movimento introdotta dalla parola "scorre" e dalla metafora "con andamento di fiume", implode nell'interruzione del predicato "si rompe" e nella disperazione che "strapiomba nel vuoto".

Qui sporgendomi da una balconata di tufo, non trapela rumore o barlume, ma mi sorprende un ribrezzo di pozzo, e con esso l'estasi che solo un irrisorio pedaggio rimanga a separarmi...Da

12 G. Bufalino, op.cit., p. 177. 
che? Non mi stancavo di domandarmelo, senza però che bastasse l'impazienza a svegliarmi;

L'Io sognante si affaccia sul silenzio buio del piccolo universo concentrazionario, dove si sorprende capace di sensazioni ossimoriche, che esita a riconoscere umane: "ribrezzo di pozzo" insinua un intenzionale immiserimento di espressioni omologhe più epiche come, ad esempio, "orrore dell'abisso"; l'associazione a "estasi" produce una efficace resa letteraria della vertigine. Lo spazio o il tempo che tengono sospeso il protagonista sul confine ("rimanga a separarmi...") non è un millimetro né un attimo, né un'inezia, ma un "irrisorio pedaggio". Un'oblazione mediante la quale espiare una colpa ignota e, con ciò, salvarsi? O solo un'interruzione della sofferenza, provvisoria liberazione dalla paura di aver paura: "Da che?", domanda che non ammette risposta accettabile, destinata, quindi, a rimanere insoddisfatta di parole.

bensì in uno stato di sdoppiata vitalità, sempre più rattratto entro le materne mucose delle lenzuola, e non per questo meno slegato ed elastico, cominciavo a calarmi di grotta in grotta, avendo per appiglio nient'altro che viluppi di malerba e schegge, fino al fondo dell'imbuto, dove, fra macerie di latomia, confusamente crescevano alberi (degli alberi non riuscivo a sognare che i nomi, ho imparato solo più tardi a incorporare nei nomi le forme). Ai piedi della scarpata, di fronte al viottolo che ne partiva, e pareva col suo rigo chiaro rassicurarmi così del repentaglio che m'ero lasciato alle spalle come dell'orridezza nuova dell'aria, esitavo un momento, in attesa che mi si calmasse nella gola il batticuore dell'avventura, e gli occhi prendessero confidenza con le visioni del sottobosco e la loro bambinesca mobilità.

Per sfuggire l'urgenza del dubbio che minaccia di traboccare dal sogno nella coscienza, l'Io scisso di malato da un lato approfondisce il suo sonno nel grembo rassicurante del 
letto, riesumando una condizione di pre-vita, di non ancora vita o, in ogni caso, di non vita, dall'altro richiama le proprie forze a nuovo vigore per esplorare il sogno in cerca di possibilità. Ma non vi trova altro che metafore della malattia: le "grotte" evocano le lesioni polmonari causate dalla TBC, gli "appigli di malerba e schegge" rappresentano l'insidia delle illusioni di remissione del male che, infine, avvelenano e frantumano la speranza. Infatti, in fondo all' "imbuto" della speranza di vita che si assottiglia, non trova che "macerie" e degli alberi solo i nomi, simulacri verbali di una realtà in dissolvenza. Il motivo dell'ostinata speranza nella cura trova una prima, misurata metafora nell'espressione "col suo rigo chiaro a rassicurarmi", sia del provvisorio scampato pericolo, sia, paradossalmente con valore di certezza, del rinnovato rischio sempre in agguato nell'elemento simbolo dei malati di tubercolosi ("orridezza nuova dell'aria").

Caduto il vento, la cui mano m'aveva a più riprese, come la mano di un complice, trattenuto o sospinto nella discesa, il silenzio era pieno; i miei passi, quelli di un'ombra. Non restava che procedere un poco, ed ecco, al posto di sempre, purgatorialmente seduti a ridosso l'uno dell'altro, uomini vestiti di impermeabili bianchi, e si scambiavano frantumi di suono, una poltiglia di sillabe balbe rimasticate in eterno da mascelle senili.

Il motivo della speranza percorre anche queste righe: nell'esplicito ricorso alla regressione all'infanzia, espressa nelle forme trepidanti dell'avventura e nella metafora del "vento" che cade, dopo aver, a più riprese, abbattuto e sostenuto; come un complice, però, non come un amico. L'atmosfera complessiva di insensatezza fin qui costruita sembra convergere nell'attribuzione di pienezza al "silenzio" opposta alla de-attribuzione (smaterializzazione) del sé dai "passi d'ombra". Un passo avanti conduce solo "nel posto 
di sempre", ogni movimento (rappresentazione del progresso nella guarigione?) viene negato immediatamente dopo esser stato formulato. La speranza nella cura è affidata ai medici dei quali l'avverbio "purgatorialmente" sancisce subito l'insufficienza, confermata dalle - altrettanto dantesche - "sillabe balbe" di una scienza dalle soluzioni balbettanti e sostanzialmente impotente ("rimasticate" e "mascelle senili"), mentre l'espressione "a ridosso l'uno dell'altro" evoca un'idea di serialità uniforme, nessuno che si distingua per valore o efficacia. Ma il periodo nel suo insieme ("purgatorialmente seduti a ridosso l'uno dell'altro") ammette, forse, anche un'altra lettura, quella di un tribunale in cui giudici-testimoni ratificano kafkianamente il castigo, rappresentato dalla malattia, di una colpa ancora ignota. Il tropo minimo degli "impermeabili bianchi" per i camici, contiene un'allusione al mito della resistenza al contagio del medico.

M'avvicinavo a loro con turbamento che l'abitudine non rendeva minore. Essi levavano mestamente la fronte, tutt'insieme accennavano un divieto, mi gridavano con spente orbite: vattene via. Non mi riusciva di obbedire, ma in ginocchio, a qualche metro di distanza, torcendomi le dita dietro la schiena, aspettavo che uno si muovesse, il più smunto, il più vecchio, una serpaia di rughe tra due lembi di bavero, e semplicemente curvandosi a raccattare una pietra, rivelasse dietro di sé, sulla soglia di un sottosuolo finora invisibile, botola di suggeritore o fenditura flegrea, la dissepolta e rapida nuca di lei, Euridice, Sesta Arduini, o come diavolo si chiamava.

"Férmati", gridavo "madre mia, ragazza, colomba", mentre sentivo il tozzo polpastrello del sonno che mi suggellava le palpebre bruscamente detumefarsi, dissiparsi in bolla di schiuma, in vischioso collirio di luce. Soltanto in quell'istante, riaprendo gli occhi, capivo d'avere ancora una volta giocato a morire, d'avere ancora una volta dimenticato, o sbagliato apposta, la parola d'ordine che serviva. 
Anche questo passo è portatore di significati complessi: il "turbamento" gerarchico di chi si accosta agli amministratori di salute e/o giustizia con la consapevolezza che nessuna vera giustizia (di vita o di diritto) sia mai possibile; l'incertezza appare confermata da "accennavano un divieto" che non è una proibizione chiara, ma un'indeterminatezza che lascia spazio all'ambiguità del sentimento dell'Io, che si manifesterà in seguito nell'idea di guarigione come tradimento, nell'oscillazione dell'anima tra un biologico istinto di sopravvivenza ed un dubbio morale di infrazione del tacito patto di non sopravvivenza tra compagni di sventura. L'ambiguità persiste nel "Non mi riusciva di obbedire" che non si risolve in atto di ribellione, ma nella resistenza passiva dell'aspettare "in ginocchio". Possibilità di disobbedienza che, infatti, arriva nelle forme del mito di Euridice (e non poteva essere altrimenti), espressione suprema delle innumerevoli e conflittuali implicazioni del rapporto amore/morte. Nell'espressione "aver ancora una volta giocato a morire" traspare un'assunzione di responsabilità oggettiva, deducibile dalla scelta del verbo giocare in luogo del corrispondente al vero sognare (il sogno non si sceglie) e confermata dall'insinuazione di "aver sbagliato apposta". La "parola d'ordine", ancora una volta, evoca un passaggio di confine. In chiusura, dunque, la meta-metafora del tradimento del sopravvissuto che è anche, probabilmente, il movente profondo del romanzo.

L'incipit di Os cus de Judas è figurativo, sembra estratto da una memoria fotografica, danneggiata da macchie di disillusione.

O que eu gostava mais no Jardim Zoológico era do rinque de patinagem sob as árvores e do professor preto muito direito a deslizar para trás no cimento em elipses vagarosas sem mover um músculo sequer, rodeado de meninas de saias curtas e botas brancas, que, se falassem, possuíam seguramente vozes tão de 
gaze como as que nos aeroportos anunciam a partida dos aviões, sílabas de algodão que se dissolvem nos ouvidos à maneira de fins de rebuçado na concha da língua.

Il dimostrativo di distanza colloca l'approccio narrativo nella dimensione del ricordo. Lo scenario è immediatamente identificabile con l'infanzia tratteggiata, per le poche righe successive, con accenti di tenerezza e levità. Al secondo posto della breve graduatoria dei lontani gradimenti dell'Io narrante, la presenza del "professor preto muito direito" propone una connotazione decisamente positiva del nero; questo motivo viene ripreso al termine del primo lungo flusso di memoria con significativo impiego di attribuzioni ("...o professor preto [..] com a majestade maravilhosa e insólita de um andor às arrecuas." p. 11): questa insistenza rimarca gli effetti tranquillizzanti della regressione ad un passato quasi edenico, caratterizzato da fratellanza e, perfino, ammirazione, in opposizione al recente, traumatico passato segnato dall' esperienza del nero come guerrigliero, nemico invisibile. Lampi di soavità ("meninas de saias curtas e botas brancas") punteggiano queste prime righe, anticipando una ricorrenza di rare ma intense illuminazioni di bellezza che attraversano tutto il romanzo, assumendo valore di pausa emotiva alla tensione verbale.

"Não sei se lhe parece idiota o que vou dizer..." La presenza di un interlocutore viene sottintesa nella forma di una dubitativa "Não sei se lhe parece idiota..."13 (nell'edizione italiana "Non so se...le sembrerà una cazzata"14 espressione leggermente più cruda rispetto al testo in lingua, ma efficace nel rendere la medesima insinuazione di un dubbio amaro di incomunicabilità, sin dalle prime battute).

13 A. Lobo Antunes, op. cit., p. 9.

14 A. Lobo Antunes, In culo al mondo, trad. it. A.Tabucchi e M.J.de Lancastre, Torino, Einaudi, 1996, p. 3. 
Ora la scena diegetica si configura animata da due personaggi. Si tratta di un punto cruciale per la critica che ha dedicato alla funzione della mulher calada, molta attenzione e qualche diversità di interpretazione. Maria Alzira Seixo sostiene che la situazione enunciativa di interlocuzione inizialmente non esiste, in termini di pragmatica del testo, essendo appena presupposta linguisticamente proprio dalla citata dubitativa. Tuttavia questa presenza viene confermata a pagina $11 \mathrm{da}$ "Se fôssemos, por exemplo, papa-formigas, a senhora e eu,..." e resa diegetica nelle successive suggestioni di intervento: "Deixe-me pagar a conta." ${ }^{15}$, "Para sua casa ou para a minha?"16, "Um pouco nu, o andar?"17, in ogni caso, la sua parola è sempre implicita in quelle dell'Io narrante. Seixo vede in questo procedimento letterario, al contempo, una derivazione ed una opposizione al discorso libero indiretto del realismo ottocentesco e lo definisce "discurso directo ocluso"18; il personaggio viene letteralmente fagocitato dal narrante che di questa appropriazione fa vivere la relazione di dialogo. L'opinione di Rui de Azevedo Teixeira al riguardo è ancora più radicale; egli sostiene che l'inesauribile capacità di ascolto della confidente è un complemento indispensabile alla logorrea del narratore ed una prova dell'impeto confessionale della scrittura, arrivando a definire Os cus de Judas: "um diálogo monologizado, no qual ao leitor, tal como à confidente de uma noite, compete apenas a função de receptáculo passivo." ${ }^{19}$.

Paulo Castilho, invece, interpreta questa modalità narrativa come la tensione del romanzo a farsi dialogo per inter-

\footnotetext{
15 A. Lobo Antunes, op. cit., p. 91 (p. 67 ed. it.).

16 Ibidem, p. 111 (p. 83 ed. it.).

17 Ibidem, p. 155 (p. 119 ed. it.).

18 M. A. Seixo, Os romances de António Lobo Antunes, Lisboa, Dom Quixote,
} 2002, p. 40.

19 R. de Azevedo Teixeira, A guerra colonial e o romance português, Lisboa, Editorial Notícias, 1998, p. 128. 
posta persona (la donna che il narratore incontra in un bar) con ciascuno dei suoi lettori. Questa metafora del rapporto Libro/Lettore mi sembra confermata dalla ostinata ricerca dell'attenzione, della comprensione, dell'approvazione della mulher calada che il narratore conduce per tutta l'opera e che costella di vere e proprie invocazioni, "Escute. Olhe para mim e escute, preciso tanto que me escute..." ${ }^{20}$, che possiamo immaginare essere il desiderio di ogni libro. Del resto, quando la donna abbandona il protagonista, il monologo si estingue, la storia finisce, proprio come quando il lettore chiude il suo libro. Ma esiste almeno un altro rapporto normalmente realizzato in una rigida e immodificabile separazione dei ruoli e attiene alla sfera del sacro. L'uomo parla con Dio e Dio tace. L'uomo gli si confessa, lo invoca, lo prega e Dio tace. E nel silenzio di Dio l'uomo costruisce da solo le proprie risposte o si rassegna a vivere facendone a meno, ma, in ogni caso, il rapporto (consolidato da una pratica in uso da circa 5000 anni) è fondato sulla separazione tra colui/colei che parla e colui/colei che ascolta. Non mi sembra un modello trascurabile.

... mas aos domingos de manhã, quando nós lá íamos com o meu pai, os bichos eram mais bichos, a solidão de esparguete da girafa assemelhava-se à de um Gulliver triste, e das lápides do cemitério dos cães subiam de tempos a tempos latidos aflitos de caniche. Cheirava aos corredores do Coliseu ao ar livre, cheios de esquisitos pássaros inventados em gaiolas de rede, avestruzes idênticas a professoras de ginástica solteiras, pinguins trôpegos de joanetes de contínuo, catatuas de cabeça à banda como apreciadores de quadros; no tanque dos hipopótamos inchava a lenta tranquilidade dos gordos, as cobras enrolavam-se em espirais moles de cagalhão, e os crocodilos acomodavam-se sem custo ao seu destino terciário de lagartixas patibulares.

${ }^{20}$ A. Lobo Antunes, op. cit., p. 71 (p. 55 ed. it.). 
La consapevolezza di ciò che comincerà a disvelarsi nel secondo capitolo, già nel passo citato corrompe il topos dell'integrità del ricordo mediante un'aggettivazione cinica e oltraggiosa, in sintonia con la strategia narrativa basata sull'ossessiva alternanza di passati e presente che si contaminano reciprocamente fino ad escludere ogni possibile purezza. Come a dire che ci sono esperienze che non lasciano intatto nulla di ciò che trovano nella vita su cui si abbattono. Così, anche l'innocente zoo fanciullesco intristisce nelle categorie sociali e morali del bestiario.

Os plátanos entre as jaulas acinzentavam-se como os nossos cabelos, e afigurava-se-me que, de certo modo, envelhecíamos juntos: o empregado de ancinho que empurrava as folhas para um balde aparentava-se, sem dúvida, ao cirurgião que me varreria as pedras da vesícula para um frasco coberto de rótulo de adesivo; uma menopausa vegetal em que os caroços da próstata e os nós dos troncos se aproximavam e confundiam irmanar-nos-ia na mesma melancolia sem ilusões; os queixais tombavam da boca como frutos podres, a pele da barriga pregueava-se de asperezas de casca. Mas não era impossível que um hálito cúmplice nos sacudisse as madeixas dos ramos mais altos, e uma tosse qualquer rompesse a custo o nevoeiro da surdez em mugidos de búzio, que a pouco e pouco adquiriam a tranquilizadora tonalidade da bronquite conjugal.

Il corpo stesso del protagonista si reifica nella metafora vegetale, valorizzata nel senso inverso a quello tradizionale - il giovane virgulto che si fa quercia - e ridotta ad una malinconica similitudine tra i nodi di un tronco d'albero e i noduli prostatici di una vecchiaia futura, già presente per l'Io straniato dall'acronia. Una vecchiaia dell'anima indifferente anche al sussulto di nuovo causato da un'improvvisa folata di vento e subito ricondotto nella piatta abitudinarietà di una 'bronquite conjugal'. 
O restaurante do Jardim, onde o odor dos animais se insinuava em farrapos diluídos no fumo do cozido, apimentando de uma desagradável sugestão de cerdas o sabor das batatas e conferindo à carne gostos peludos de alcatifa, encontrava-se ordinariamente repleto, em doses equitativas, de grupos excursionistas e de mães impacientes, que afastavam com os garfos balões à deriva como sorrisos distraídos, a arrastarem pontas de guita atrás de si, tal as noivas volantes de Chagall a bainha dos vestidos. Senhoras idosas vestidas de azul, com tabuleiros de bolos na barriga, ofereciam travesseiros mais poeirentos do que as suas bochechas folhadas, perseguidas pelo fastio pegajoso das moscas. Cães esqueléticos de retábulo medieval hesitavam entre a biqueira dos empregados e as salsichas que sobravam dos pratos para o chão à laia de dedos supérfluos, luzidios da brilhantina do óleo.

Nella descrizione del ristorante e dei suoi frequentatori si respira l'aria maleodorante di un girone dantesco, periferico e purgatoriale, confermato dalla presenza sintagmatica di maiale e moquette, entrambi sudici involontariamente, privi, cioè, anche della dignità di esserlo per scelta. Il motivo della non scelta per incapacità, cecità, codardia, qui implicito al livello lessicale, viene sviluppato tormentosamente lungo tutta la narrazione. L'estensione ironica di Lobo Antunes risulta quasi sconfinata, è una dura roccia su cui egli spunta tutte le armi della retorica fino a ricomporne una di segno opposto, dal senso rovesciato, con funzione antidotica al rischio, in egual misura temuto e perseguito, di pietismo e commiserazione. È all'ironia che l'autore affida la reiterata insinuazione di bassezza, di disvalore, di turpitudine, fino a formulare una sorta di avvertenza permanente: in questa storia non ci sono eroi, la dolcezza del vivere non è mai un sentimento, tutt'al più un minuscolo mosaico di piccoli fatti e ricordi sbiaditi, lo strazio e il dolore stentano a superare l'epicità di una colica renale. La materia della narrazione è immonda, gretta, assurda, intenzionalmente anti-mitica. 
Os cus de Judas è suddiviso in ventitrè capitoli, quante sono le lettere dell'alfabeto portoghese, ciascuno dei quali dotato di una struttura autosufficiente, ragione per la quale gli incipit sono in numero pari ai capitoli. Quindici aprono in forma dialogica; ciò può essere considerato un indizio dell'intenzione narrativa profonda: il dialogo come tentativo di ristrutturazione dell'umano stravolto dall'irruzione dell'assurdo nella vita.

Un'ultima osservazione a proposito dei due incipit. Giuseppe Amoroso, occupandosi di Diceria dell'untore, nota che “...quella sottolineatura degli uomini come spettatori inetti di se stessi e dell'esterno, e del personaggio come un pugno di spine dure, reca una intercambiabilità di misure rappresentative che può provocare un inedito sbocco di incontri tra elementi della sfera umana e elementi dell'ambiente..."21 che mi sembra corrispondere a quanto si è notato a proposito di alcune metafore contenute nel brano di Os cus de Judas riportato (la menopausa vegetal $=$ il ribrezzo di pozzo).

3) Il sentimento di ineluttabilità della sconfitta permea le espressioni rappresentative dei due romanzi: "doloroso apprendistato di morte" per la Diceria dell'untore e "dolorosa aprendizagem da agonia" per il romanzo di Lobo Antunes.

Il decorso lessicale è comune fino all'epilogo, in cui si differenzia per il carattere definitivo e irrimediabile di "morte" rispetto ad "agonia" che ancora trattiene un residuo di sommessa e diffidente speranza, ma, anche, di prolungamento del dolore. Entrambi i romanzi mi sembrano pervasi da un sentimento di rivolta, fosse anche solo contro la dimenticanza o la rimozione; in quello di Bufalino si disperde nelle forme di una leopardiana lucidità, confermata dal finale, linguisticamente raggelato in una serie di imper-

21 G. Amoroso, Narrativa italiana 1975-1983, Milano, Mursia, 1983, p. 249. 
fetti che preludono a un futuro imperfetto, privo di aspettative e di sorprese:

M'aspettava una vita nuda, uno zero di giorni previsti, senza una brace né un grido. Uscire mi toccava dalla cruna dell'individuo per essere uno dei tanti della strada, che amministrano umanamente la loro piccina saviezza d'alito e d'anni.[...] Per questo forse m'era stato concesso l'esonero; per questo io solo m'ero salvato, e nessun altro, dalla falcidia: per rendere testimonianza, se non delazione, d'una retorica e d'una pietà. Benché sapessi già allora che avrei preferito starmene zitto e portarmi lungo gli anni la mia diceria al sicuro sotto la lingua, come un obolo di riserva, con cui pagare il barcaiolo il giorno in cui mi fossi sentito, in séguito ad altra e meno remissibile scelta o chiamata, sulle soglie della notte. ${ }^{22}$

Nel protagonista di Os cus de Judas, invece, il sentimento di rivolta sembra declinarsi in una camusiana revoca della propria emotività dal mondo. Sotto questa luce, le reiterate autoaccuse di incapacità d'amore, di inettitudine ai rapporti affettivi, di inadeguatezza fisica che, lungo tutta la narrazione, contrappuntano le accuse alla politica, alla società portoghese bigotta, ai membri della famiglia, possono aprire un nuovo spazio di significato: una volontaria mutilazione della propria umanità.

A sua volta questa ipotesi si biforca in due linee d'interpretazione:

a) una presa di distanza da un mondo irrimediabilmente privo di senso e oscenamente dedito alla violenza; non un semplice dissenso intellettuale da coltivare, tutto sommato a buon mercato, sulle rive ospitali della Senna (" ... queria achar-me em Paris a fazer revoluções no café..."23), ma un allontanamento esistenziale, il cui costo elevato si paga di persona;

22 G. Bufalino, op. cit., p. 133.

23 A. Lobo Antunes, op. cit., p. 201(p. 156 ed. it.). 
b) mutilazione come effetto dell'ordalia, castigo estremo per la propria impotenza ad opporsi per cambiare le cose.

Ma l'infamia del tempo di Lobo Antunes non ha la grandezza di quella del tempo di Camus, così anche la revoca dell'emotività del suo personaggio non raggiunge la nettezza sovrumana dello Straniero, ma finisce con l'ammettere una possibilità di patteggiamento, una modesta ipotesi di futuro:

Eu? Fico ainda mais um bocado por aqui. Vou despejar os cinzeiros, lavar os copos, dar um arranjo à sala, olhar o rio. Talvez volte para a cama desfeita, puxe os lençóis para cima e feche os olhos. Nunca se sabe, não é?, mas pode bem acontecer que a tia Teresa me visite." ${ }^{24}$

Un'ultima considerazione sul finale: quello di Os cus de Judas si colloca cronologicamente nel mattino, quello della Diceria dell'untore chiude con la parola notte.

4) Walter Benjamin ha scritto che l'unica realtà conoscibile è la superficie. La superficie dei due romanzi è lo stile, lussureggiante, ossessivo, barocco, declinato nelle due varianti del lirismo e dell'espressionismo:

...il registro alto, lo scialo degli aggettivi, l'oltranza dei colori, mi pareva, e mi pare, il modo che ci resta per contrastare l'ossificazione del mondo in oggetti senza qualità e per restituire ai nostri occhi ormai miopi il sangue forte delle presenze e dei sentimenti. ${ }^{25}$

Il discorso in prima persona, il lungo monologo del protagonista sarebbe potuto diventare difficilmente sopportabile per il lettore se questi non fosse continuamente stimolato dalla successione straripante di aggettivazioni crudeli e incisive, di

\footnotetext{
24 Ibidem, p. 244 (p. 192 ed. it.).

25 G. Bufalino, op. cit, p.178.
} 
paragoni, metafore, immagini sorprendenti, dalla verbosità lussuriosa che costituisce la marca principale dello stile di Lobo Antunes. ${ }^{26}$

Gesualdo Bufalino, che ragiona sul suo testo, lo glossa, lo analizza, lo anatomizza, lo disvela al lettore fornendo il repertorio delle singole strutture sintattiche, semantiche, mitiche e tematiche, dice del suo linguaggio figurato: 'La metafora è il cibo della mia prosa, e non starò a giustificarmene o a vantarmene. È un procedimento tipicamente barocco, anche se nel mio caso io parlerei di barocco borrominiano nel quale l'ornato è una funzione, senza di esso la struttura cadrebbe. "27 Bufalino è estremamente consapevole delle armi che adopera per restituire ad occhi miopi "il sangue forte della presenza e del sentimento" 28 .

Il titolo Os cus de Judas riproduce, in declinazione plurale, un idiotismo che ha significato equivalente all'italiano casa del diavolo (che, peraltro, esiste in identica formulazione in portoghese) di cui o $\mathrm{cu}$ de Judas può essere considerata una variante volgare, più violenta, con un'indubbia implicazione di risentimento e rabbia. Arlindo Castanho ${ }^{29}$ considera alcune delle probabili origini dell'espressione, finendo col propendere per il versetto 20 del primo capitolo del libro degli Atti: "Infatti sta scritto nel libro dei Salmi: La sua dimora diventi deserta e nessuno vi abiti". Egli rinviene nella vitalità di questo stigma di luogo abominevole nella fantasia popolare, la matrice della ferocemente eloquente espressione o cu de Judas che i portoghesi riferiscono agli angoli più remoti ed inospitali della Terra.

26 A. Castanho, in AAVV, Dalle armi ai garofani- Studi sulla letteratura della guerra coloniale-, a cura di Manuel G. Simões e R. Vecchi, Roma, Bulzoni, 1995, p. 31.

27 F. Caputo, "Prefazione" in G.Bufalino, op. cit., p. VI.

28 Ibidem, p. 178.

29 A. Castanho, op. cit., p. 31. 
Vorrei tornare sul numero grammaticale che direi sintomo di una superiore semantica testuale: $o c u$ de Judas del protagonista non è soltanto Luanda o il Nordest angolano, ma è il Portogallo (quello di Salazar certamente, ma anche quello post 25 aprile che, dopo un iniziale scatto politico e morale, gli sembra ristagnare inerte, irrisolto), è il perbenismo asfissiante che ha intriso la sua infanzia, è l'occlusione sentimentale che lo relega ad una solitudine umiliante. Dunque, un'inospitalità plurale.

Bufalino, critico di se stesso, fornisce la sua consapevole chiave di lettura del titolo: "Diceria vale racconto, dettato, monologo con in più una insinuazione di scarsa credibilità, come di uno sproloquio mormorato all'orecchio." ${ }^{30}$. E, indirettamente, conferma la vicinanza a Os cus de Judas. Infatti l'insinuazione di scarsa credibilità mina la solidità della costruzione narrativa in quanto rappresentazione estetica di un vissuto esperienziale che i due autori, ed il lettore con essi, sentono assurdo e, quindi, non credibile; la malattia e la guerra sono picchi di insensatezza particolarmente inaccettabili.

Da questa situazione limite, entrambi derivano l'estrema opzione esistenziale, che attraversano lucidamente e con quieto sgomento: l'abiezione.

Non si vedeva, e io pensavo, con un acido orrido aculeo di bramosia, alle sue membra emissarie di umori, ai suoi sputi, colaticci, sudori, lacrime, essudati, ai suoi profluvi d'emorroissa dannata, alle sue emottisi trionfali. Che strano innamorarsi di un corpo che mangia, secerne, si svuota: denso di villi, papille, isole del Malpighi... Nomi [...] che ripetevo ora, recuperandoli al disopra del frastuono degli anni, per servirmene a investigare la geologia di quell'umido sepolcro di carne... ${ }^{31}$

${ }^{30}$ G. Bufalino, op. cit., p. 178.

31 Ibidem, p. 78. 
Entrei no quarto de banho dos sargentos, na pocilga eternamente inundada e nauseabunda a que se chamava quarto de banho dos sargentos [...] As nádegas do homem formavam um movimento de êmbolo que se apressava, a camisa pegavase às costas em ilhas imprecisas de suor, o queixo tremia como o dos reformados nos refeitórios dos asilos, as pupilas ocas da prisioneira miravam-me numa fixidez insuportável, e apeteceu-me, entende, tirar também a minha pila para fora e urinar sobre eles... ${ }^{32}$

La fascinazione dell'oralità, l'urgenza della testimonianza, l'astioso piacere del reduce nel raccontare e la vergogna di essere sopravvissuto, sono caratteristiche comuni a questi e ad un altro grande, scomodo, romanzo: Voyage au bout de la nuit. Vent'anni dopo la pubblicazione del Voyage, Céline confessò in un'intervista: "c'erano delle interdizioni, uno steccato riservato. Questo steccato l'ho superato senza saperlo, correndo dietro il tono giusto, il movimento vero, nella loro forma più espressiva" 33 .

Sembra proprio la descrizione di una sorta di resistenza alla comunicazione di alcuni temi che solo il trattamento artistico riesce a vincere. Con ciò sottraendo alla dimenticanza quell'insostenibile umano troppo umano che appartiene a ognuno di noi.

32 A Lobo Antunes, op. cit., p.221 (p.172 ed.it.).

33 L. F. Céline, Viaggio al termine della notte, Milano, Corbaccio, 1998. 